\title{
Energy momentum tensor for translation invariant renormalizable noncommutative field theory
}

\author{
Ezinvi Baloïtcha ${ }^{1, \mathrm{a}}$, Vincent Lahoche ${ }^{2, \mathrm{~b}}$, and Dine Ousmane Samary ${ }^{1,3, \mathrm{c}}$ \\ ${ }^{1}$ Faculté des Sciences et Techniques, International Chair in Mathematical Physics and Applications (ICMPA-UNESCO Chair), \\ University of Abomey-Calavi, 072B.P.50, Cotonou, Benin \\ ${ }^{2}$ LaBRI, Univ.Bordeaux 351 cours de la Libération, 33405 Talence, France \\ 3 Max Planck Institute for Gravitational Physics, Albert Einstein Institute, Am Mühlenberg 1, 14476, Potsdam, Germany
}

Received: 12 July 2018 / Revised: 7 October 2018

Published online: 14 December 2018

(C) The Author(s) 2018. This article is published with open access at Springerlink.com

\begin{abstract}
In this paper, we derive the energy momentum tensor for the translation invariant noncommutative Tanasa et al. scalar field model. The Wilson regularization procedure is used to improve this tensor and the local conservation property is recovered. The same question is addressed in the case where the Moyal star product is deformed including the tetrad fields. It provides us with an extension of the recent work (J. Phys. A: Math. Theor. 43, 155202 (2010)), regarding the computation and properties of the Noether currents to the renormalizable models.
\end{abstract}

\section{Introduction}

Noncommutative (NC) geometry and its applications to quantum field theory (QFT), namely NCQFT, has received increasing attention, in the two decades, due to the advent of the class of renormalizable actions [1-11]. NCQFT arises as a scenario for the Planck scale behavior of physical theories, at which the nonlocality of interactions has to appear and break down the notion of continuous spacetime $[12,13]$. It is most often performed over a Moyal space $\mathbb{R}_{\theta}^{d}$. This space is the deformation of $d$-dimension Euclidean space $\mathbb{R}^{d}$ endowed with a constant Moyal product of functions:

$$
(f \star g)(x)=\mathbf{m}\left\{e^{i \frac{i^{\rho \sigma}}{2} \partial_{\rho} \otimes \partial_{\sigma}} f(x) \otimes g(x)\right\}, \quad x \in \mathbb{R}^{d}, f, g \in C^{\infty}\left(\mathbb{R}^{d}\right),
$$

where $\mathbf{m}(f \otimes g)=f \cdot g$, and such that the coordinates functions $x^{\sigma}$ and $x^{\rho}$ satisfy the commutation relation

$$
\left[x^{\rho}, x^{\sigma}\right]_{\star}=i \theta^{\rho \sigma} .
$$

$\theta^{\rho \sigma}$ is a skew symmetric constant tensor and elements have the dimension of the length square. It is possible to construct the NCQFT in a nontrivial background metric, generally by imposing the nonconstant deformation matrix $\theta^{\rho \sigma}=\theta^{\rho \sigma}(x)$, which naturally results in the difficulty of finding a suitable explicit closed Moyal-type formula [14-18]. In the context of a dynamical NC field theory, this can be realized by replacing the vector field $\partial_{\mu}$ on the tangent space $T_{x} \mathbb{R}_{\theta}^{d}$ by $X_{a}=e_{a}^{\mu}(x) \partial_{\mu}$, where the tetrad $e_{a}^{\mu}(x)$ is a tensor depending on the coordinate functions. The generalized Moyal star-product becomes

$$
(f \star g)(x)=\mathbf{m}\left\{e^{i \frac{\theta^{a b}}{2} X_{a} \otimes X_{b}} f(x) \otimes g(x)\right\}, \quad x \in \mathbb{R}^{d}, f, g \in C^{\infty}\left(\mathbb{R}^{d}\right) .
$$

In general, the vector field $X_{a}$ is noncommutative with respect to the Lie bracket " $[\cdot, \cdot]$ ". The particular condition $\left[X_{a}, X_{b}\right]=0$ results in constraints on the tetrad $e_{a}^{\mu}$ and leads to the definition of one new field $\varphi^{a}$, such that the inverse $e_{\nu}^{a}$ of $e_{a}^{\nu}$ is proportional to $\partial_{\nu} \varphi^{a}$. Since $X_{a} \varphi^{b}=\delta_{a}^{b}$, the field $\varphi^{b}$ can be viewed as new coordinates along the

\footnotetext{
a e-mail: ezinvi.baloitcha@cipma.uac.bj

b e-mail: vincent.lahoche@labri.fr

c e-mail: ousmanesamarydine@yahoo.fr
} 
$X_{a}$-directions and therefore will be taken into account in the redefinition of the functional action [16]. The Moyal space $\mathbb{R}_{\theta}^{d}$ of this type becomes curved with the background metric $\mathrm{g}_{\mu \nu}=e_{\mu}^{a} e_{\nu}^{b} \delta_{a b}$. Let us mention that the commuting vector field $X_{a}$ ensures the associativity of the star product (3), but the loss of the associativity property becomes evident in the general case where $\left[X_{a}, X_{b}\right] \neq 0$. Nevertheless, this property may be satisfied in a space with a nearly Euclidean metric, in which it is natural to choose a tetrad field $e_{a}^{\mu}(x)$ that lies nearly along the coordinate axes $e_{a}^{\mu}(x)=\delta_{a}^{\mu}+\omega_{a}^{\mu}(x)$, where $\omega_{a}^{\mu}(x)$ is a coordinate-dependent small quantity to be determined [19,20].

The basic problem which has accompanied the development of NC field theory is the UV-IR mixing in the perturbation computation [21]. This pathology may be solved by introducing in the scalar field action, i.e. the $\varphi_{\star}^{4}$-model, the so-called Grosse-Wulkenhaar (GW) harmonic term [3-5]. The GW model breaks the $U(N)$ symmetry invariance in the IR regime but is asymptotically safe in the UV regime. The model is also noninvariant under the translation and rotation of the spacetime. The only known invariance satisfied by the model is the so-called Langman-Szabo duality [22]. The study of the symmetry consequence, such as the Noether current, are addressed for the GW model by imposing a constraint on the Euler-Lagrange (EL) equations of motion [23-25]. In [26] the same question is addressed in the case of twisted star product definition in the field theory.

Using the same idea of the perturbative computation of the renormalization procedure, other theoretical model have been proved to be renormalizable. The theoretical ingredient to perform this issue is the so-called multiscale analysis, developed by Rivasseau [28]. One of the models we will focus on in this work is the translation invariant renormalizable scalar model discovered by Gurau-Magnen-Rivasseau-Tanasa (GMRT) [7-11]. The GMRT model comes from the NC $\varphi^{4}$ model by adding a new contribution $\frac{a}{\theta^{2} p^{2}}$ on the propagator in the momentum space, and on which the problem of UV/IR mixing is solved. At any order in perturbation theory, the $\beta$ functions of the model are given [27]. Despite all these interesting results, the corresponding current derived from the symmetry properties of the Tanasa model has not yet been given in the literature. Our purpose in this paper is to investigate the computation of the energy momentum tensor (EMT) of the GMRT model and study its regularization in both ordinary Moyal space and twisted Moyal space. The Wilson regularization procedure is used to recover the local conservation as given in [29-37].

The paper is organized as follows. In sect. 2 we compute the EMT for translation invariant GMRT model in the ordinary Moyal space. The regularization of this tensor is also given. In sect. 3 the same computation is performed in the case of the twisted Moyal plane. Our conclusion and remarks are given in sect. 4 .

\section{EMT for the renormalizable GMRT model in Moyal space}

In this section we derive the EL equation of motion and the EMT for the translation invariant nonlocal functional action. The renormalization procedure described in [9] is also pointed out. Let us consider the scalar field theoretic model in which we begin with the Lagrange density, which is a function of the $\varphi$ field, its first partial spacetime derivatives $\partial_{\mu} \varphi$, and the inverse derivatives $\partial_{\mu}^{-1} \varphi$ :

$$
S_{\star}[\varphi]=\int \mathrm{d}^{d} x \mathcal{L}_{\star}\left(\varphi, \partial_{\mu} \varphi, \partial_{\mu}^{-1} \varphi\right), \quad \partial_{\mu}^{-1} \varphi(x):=\int_{-\infty}^{x} \mathrm{~d} x^{\prime \mu} \varphi\left(x^{\prime}\right),
$$

where $\mathcal{L}_{\star}$ means that the ordinary product of function in the action $S$ is replaced by the Moyal product, i.e.

$$
\mathcal{L}_{\star}\left(\varphi, \partial_{\mu} \varphi, \partial_{\mu}^{-1} \varphi\right)=\mathcal{L}\left(\varphi, \partial_{\mu} \varphi, \partial_{\mu} \partial_{\nu} \varphi, \partial_{\mu} \partial_{\nu} \partial_{\sigma} \varphi, \cdots \infty, \partial_{\mu}^{-1} \varphi\right) .
$$

Before starting our investigation on the computation of the EMT, let us provide the following important remark on the Lagrangian density (5). First of all, the noncommutative fields theories are nonlocal in time and space due to an infinite number of temporal and spatial derivatives in the Lagrangian. This infinite number of derivatives comes from the definition of the star product. Despite the fact that the inverse derivative $\partial_{\mu}^{-1} \varphi$ is considered as a nonlocal contribution in the Lagrangian (5), this Lagrangian remains nonlocal without this contribution. The analysis performed in this work concerning the computation of the EMT becomes similar to what follows in the previous literature, concerning the Noether current applied to noncommutative fields theories (see [16, 23-26, 29-37] and references therein).

Under the translation group, which transforms coordinates as $x^{\mu} \rightarrow x^{\mu}+a^{\mu}$ ( $a^{\mu}$ is a constant vector), the $\varphi$ field is then transformed as $\varphi(x) \rightarrow \varphi(x)+a^{\mu} \partial_{\mu} \varphi(x)$; the variation of action (4) gives

$$
S_{\star}[\varphi] \rightarrow S_{\star}[\varphi]+\delta S_{\star}[\varphi]=S_{\star}[\varphi]+a^{\mu} \partial_{\mu} S_{\star}[\varphi],
$$

where we have assumed that the $\varphi$ field vanishes when $|x|$ approaches infinity. We will show that the translation invariance of the nonlocal action, $\delta S_{\star}[\varphi]=0$, implies the existence of a conserved current density $J_{\nu}$, such that

$$
\delta \varphi \star \frac{\delta S_{\star}}{\delta \varphi}+\partial^{\nu} J_{\nu}=0
$$


where the infinitesimal current $J_{\nu}$ may be expressed in terms of the EMT as

$$
\int \mathrm{d}^{d} x \partial_{\mu} J^{\mu}:=a^{\nu} \int \mathrm{d}^{d} x \partial_{\mu} T_{\nu}^{\mu}
$$

Note that the above relation is well satisfied if $\partial^{\alpha} \varphi \in \mathcal{S}\left(\mathbb{R}^{d}\right), \alpha=\llbracket-2,-1 \rrbracket \cup \mathbb{N}$, where $\mathcal{S}\left(\mathbb{R}^{d}\right)$ is the space of suitable Schwartzian functions. The variation of the action $S_{\star}$ is written as

$$
\delta S_{\star}=\int \mathrm{d}^{d} x\left[\frac{\partial \mathcal{L}_{\star}}{\partial \varphi} \star \delta \varphi+\frac{\partial \mathcal{L}_{\star}}{\partial \partial_{\mu} \varphi} \star \partial_{\mu} \delta \varphi+\frac{\partial \mathcal{L}_{\star}}{\partial \partial_{\mu}^{-1} \varphi} \star \partial_{\mu}^{-1} \delta \varphi\right] .
$$

It turns out that the inverse derivative, which appears in (4), is such that $\partial_{\mu} \partial_{\nu}^{-1} \varphi(x)=\delta_{\mu \nu} \varphi(x)$ and $\partial_{\mu}(a b)=$ $\left(\partial_{\mu} a\right) b+a\left(\partial_{\mu} b\right)$. Then, the following identity is well satisfied:

$$
\sum_{\mu=1}^{d} \frac{\partial \mathcal{L}_{\star}}{\partial \partial_{\mu}^{-1} \varphi} \star \partial_{\mu}^{-1} \delta \varphi=\sum_{\mu=1}^{d} \partial_{\mu}\left[\partial_{\mu}^{-1}\left(\frac{\partial \mathcal{L}_{\star}}{\partial \partial_{\mu}^{-1} \varphi}\right) \star \partial_{\mu}^{-1} \delta \varphi\right]-\sum_{\mu=1}^{d} \partial_{\mu}^{-1}\left(\frac{\partial \mathcal{L}_{\star}}{\partial \partial_{\mu}^{-1} \varphi}\right) \star \delta \varphi .
$$

Note that this relation comes from the Leibniz rule. In the rest of this work, without all confusions the repetitive "double indices" is summed as Einstein summation. In the case of repetitive "triple indices" or more, the Einstein summation is not satisfied. If this is not the case, we will specify if this summation holds. Thus, the EL equations of motion for the Lagrangian density $\mathcal{L}_{\star}$ become

$$
E_{\varphi}=\frac{\partial \mathcal{L}_{\star}}{\partial \varphi}-\partial_{\mu}\left(\frac{\partial \mathcal{L}_{\star}}{\partial \partial_{\mu} \varphi}\right)-\partial_{\mu}^{-1}\left(\frac{\partial \mathcal{L}_{\star}}{\partial \partial_{\mu}^{-1} \varphi}\right)=0,
$$

and the conserved EMT can be derived by replacing, in relation (7), $\delta \varphi$ by $-a^{\mu} \partial_{\mu} \varphi$, such that

$$
\int \mathrm{d}^{d} x\left(-a^{\rho} \partial^{\mu} T_{\mu \rho}+E_{\varphi}\right)=0,
$$

where

$$
T_{\mu \rho}=\frac{1}{2}\left\{\frac{\partial \mathcal{L}_{\star}}{\partial \partial_{\mu} \varphi}, \partial_{\rho} \varphi\right\}_{\star}+\frac{1}{2}\left\{\partial_{\mu}^{-1}\left(\frac{\partial \mathcal{L}_{\star}}{\partial \partial_{\mu}^{-1} \varphi}\right), \partial_{\mu}^{-1} \partial_{\rho} \varphi\right\}_{\star}-g_{\mu \rho} \mathcal{L}_{\star} .
$$

Consider, as an example, the translation invariant noncommutative field theory [8,9], which is proposed to take into account the quantum corrections and to avoid the UV/IR mixing of the noncommutative scalar field theory:

$$
S_{\star}[\varphi]=\int \mathrm{d}^{d} x\left[\frac{1}{2} \partial_{\mu} \varphi \star \partial^{\mu} \varphi+\frac{m^{2}}{2} \varphi \star \varphi+\frac{a}{2 \theta^{2}} \partial_{\mu}^{-1} \varphi \star \partial_{\mu}^{-1} \varphi+\frac{\lambda}{4 !} \varphi_{\star}^{4}\right],
$$

where

$$
\partial_{\mu}^{-1} \varphi(x):=\int_{-\infty}^{x} \mathrm{~d}^{\mu} x^{\prime} \varphi\left(x^{\prime}\right), \quad \int_{-\infty}^{x} \frac{\delta \varphi\left(x^{\prime}\right)}{\delta \varphi(y)} \mathrm{d} x^{\prime}=\Theta(x-y),
$$

$\Theta(x)$ is the Heaviside function. The following statement holds.

Proposition 1. The GMRT functional action (14) is invariant under spacetime translation. This symmetry implies the global conservation of the tensor (13) due to relation (12). Moreover the tensor (13) leads to the construction of a symmetric and locally conserved EMT given by

$$
\begin{aligned}
T_{\rho \mu}^{s, r}= & \frac{1}{2}\left\{\partial_{\mu} \varphi, \partial_{\rho} \varphi\right\}_{\star}+\frac{a}{4 \theta^{2}}\left\{\partial_{\mu}^{-1} \partial_{\mu}^{-1} \varphi, \partial_{\mu}^{-1} \partial_{\rho} \varphi\right\}_{\star}+\frac{a}{4 \theta^{2}}\left\{\partial_{\rho}^{-1} \partial_{\rho}^{-1} \varphi, \partial_{\rho}^{-1} \partial_{\mu} \varphi\right\}_{\star} \\
& -i \frac{\lambda}{4 !} \theta^{\alpha \beta} g_{\alpha \rho}\left(\left[\partial_{\mu} \varphi, \varphi\right]_{\star} \star^{\prime} \partial_{\beta}(\varphi \star \varphi)\right)-i \frac{\lambda}{4 !} \theta^{\alpha \beta} g_{\alpha \mu}\left(\left[\partial_{\rho} \varphi, \varphi\right]_{\star} \star^{\prime} \partial_{\beta}(\varphi \star \varphi)\right) \\
& -g_{\mu \rho} \mathcal{L}_{\star},
\end{aligned}
$$

where we have introduced the new star product $\star^{\prime}$, which is nonassociative and commutative and is given by

$$
f \star^{\prime} g=\mathbf{m}\left\{\frac{\sin \left(\frac{1}{2} \theta^{\mu \nu} \partial_{\mu} \otimes \partial_{\nu}\right)}{\frac{1}{2} \theta^{\mu \nu} \partial_{\mu} \otimes \partial_{\nu}}(f \otimes g)\right\} .
$$


Proof. The variation principle gives the EL equations of motion (see eq. (11) for more details):

$$
\frac{\delta S_{\star}}{\delta \varphi}=0 \Leftrightarrow-\partial_{\mu} \partial_{\mu} \varphi+m^{2} \varphi+\frac{\lambda}{3 !} \varphi_{\star}^{3}-\frac{a}{\theta^{2}} \partial_{\mu}^{-1} \partial_{\mu}^{-1} \varphi=0 .
$$

Furthermore the EMT becomes

$$
T_{\mu \rho}=\frac{1}{2}\left\{\partial_{\mu} \varphi, \partial_{\rho} \varphi\right\}_{\star}+\frac{a}{2 \theta^{2}}\left\{\partial_{\mu}^{-1} \partial_{\mu}^{-1} \varphi, \partial_{\mu}^{-1} \partial_{\rho} \varphi\right\}_{\star}-g_{\mu \rho} \mathcal{L}_{\star} .
$$

The tensor $T_{\mu \rho}$ is nonsymmetric and nonlocally conserved. Let $T_{\mu \rho}^{s}$ be the symmetric tensor associated to $T_{\mu \rho}$, i.e., $T_{\mu \rho}^{s}=\left(T_{\mu \rho}+T_{\rho \mu}\right) / 2$, we get

$$
T_{\mu \rho}^{s}=\frac{1}{2}\left\{\partial_{\mu} \varphi, \partial_{\rho} \varphi\right\}_{\star}+\frac{a}{4 \theta^{2}}\left\{\partial_{\mu}^{-1} \partial_{\mu}^{-1} \varphi, \partial_{\mu}^{-1} \partial_{\rho} \varphi\right\}_{\star}+\frac{a}{4 \theta^{2}}\left\{\partial_{\rho}^{-1} \partial_{\rho}^{-1} \varphi, \partial_{\rho}^{-1} \partial_{\mu} \varphi\right\}_{\star}-g_{\mu \rho} \mathcal{L}_{\star} .
$$

Note that the procedure of regularization of the EMT performed by Gerhold et al. [29] can be used. Consider the star product $\star^{\prime}$ given in (17), which satisfies the following identity:

$$
\theta^{\mu \nu} \partial_{\mu} f \star^{\prime} \partial_{\nu} g=-i[f, g]_{\star}
$$

Then, after little computation, we get

$$
\partial^{\rho} T_{\rho \mu}^{s}=\frac{\lambda}{4 !}\left[\left[\partial_{\mu} \varphi, \varphi\right]_{\star}, \varphi \star \varphi\right]_{\star}=i \frac{\lambda}{4 !} \theta^{\alpha \beta} \partial_{\alpha}\left(\left[\partial_{\mu} \varphi, \varphi\right]_{\star} \star^{\prime} \partial_{\beta}(\varphi \star \varphi)\right) .
$$

The locally conserved EMT then becomes

$$
\begin{aligned}
T_{\rho \mu}^{s, r}= & \frac{1}{2}\left\{\partial_{\mu} \varphi, \partial_{\rho} \varphi\right\}_{\star}+\frac{a}{4 \theta^{2}}\left\{\partial_{\mu}^{-1} \partial_{\mu}^{-1} \varphi, \partial_{\mu}^{-1} \partial_{\rho} \varphi\right\}_{\star}+\frac{a}{4 \theta^{2}}\left\{\partial_{\rho}^{-1} \partial_{\rho}^{-1} \varphi, \partial_{\rho}^{-1} \partial_{\mu} \varphi\right\}_{\star} \\
& -i \frac{\lambda}{4 !} \theta^{\alpha \beta} g_{\alpha \rho}\left(\left[\partial_{\mu} \varphi, \varphi\right]_{\star} \star^{\prime} \partial_{\beta}(\varphi \star \varphi)\right)-i \frac{\lambda}{4 !} \theta^{\alpha \beta} g_{\alpha \mu}\left(\left[\partial_{\rho} \varphi, \varphi\right]_{\star} \star^{\prime} \partial_{\beta}(\varphi \star \varphi)\right) \\
& -g_{\mu \rho} \mathcal{L}_{\star} .
\end{aligned}
$$

Note that the limit $a \rightarrow 0$ gives the EMT for the scalar field theory on Moyal space derived in [29,30], from which the Belifante PDE can be given. Also by adding the quantity $\frac{1}{6}\left(g_{\mu \rho} \square-\partial_{\mu} \partial_{\rho}\right)(\varphi \star \varphi)$ in expression (20) and by setting $m=0$, we obtain the traceless EMT. The conventional tensor (19) does not have finite matrix elements even to lowest order in the coupling $\lambda$. However, the modified tensor $T_{\mu \rho}^{I}=T_{\mu \rho}-\frac{1}{6}\left(g_{\mu \rho} \square-\partial_{\mu} \partial_{\rho}\right)(\varphi \star \varphi)$ has finite matrix elements to all orders in $\lambda$. The improvement term does not contribute to the divergence of the energy-momentum tensor, i.e.

$$
\partial_{\mu} T_{\mu \rho}^{I}=\partial_{\mu} T_{\mu \rho}
$$

The global conservation of the EMT (16) implies the existence of a conserved $d$-momentum $P_{\rho}$, such that

$$
\partial^{0} P_{\rho}=\partial^{0} \int \mathrm{d}^{d} x T_{0 \mu}^{s, r}=0 .
$$

Due to the presence of the deformation parameter $\theta^{\mu \nu}$ (as a constant, skewsymmetric, fixed tensor), the Lorentz symmetry is manifestly broken. In the next section we introduce a deformation of the Moyal algebra, so-called dynamical Moyal algebra, in which the tensor $\theta^{\mu \nu}$ depends now on the coordinates. In this situation the Lorentz symmetry maybe restored and therefore the corresponding EMT becomes Lorentz invariant tensor. Consider the tensor $x_{\rho \mu}(a, \theta)$ given by

$$
x_{\rho \mu}(a, \theta)=\frac{a}{4 \theta^{2}}\left\{\partial_{\mu}^{-1} \partial_{\mu}^{-1} \varphi, \partial_{\mu}^{-1} \partial_{\rho} \varphi\right\}_{\star}+\frac{a}{4 \theta^{2}}\left\{\partial_{\rho}^{-1} \partial_{\rho}^{-1} \varphi, \partial_{\rho}^{-1} \partial_{\mu} \varphi\right\}_{\star} .
$$

The indices $\rho$ and $\mu$ are not summed in the right-hand side of (26). Also we can use the fact that $\partial_{\mu}^{-1} \partial_{\rho} \varphi=\delta_{\mu \rho} \varphi$ and, therefore, $x_{\rho \mu}(a, \theta)$ becomes

$$
\begin{aligned}
x_{\rho \mu}(a, \theta) & =\frac{a}{4 \theta^{2}}\left\{\partial_{\mu}^{-1} \partial_{\mu}^{-1} \varphi, \varphi\right\}_{\star}+\frac{a}{4 \theta^{2}}\left\{\partial_{\rho}^{-1} \partial_{\rho}^{-1} \varphi, \varphi\right\}_{\star} \\
& =\frac{a}{4 \theta^{2}}\left\{\int \mathrm{d}^{\mu} x\left(\int \mathrm{d}^{\mu} x \varphi\right), \varphi\right\}_{\star}+\frac{a}{4 \theta^{2}}\left\{\int \mathrm{d}^{\rho} x\left(\int \mathrm{d}^{\rho} x \varphi\right), \varphi\right\}_{\star} .
\end{aligned}
$$

Furthermore the tensor $t_{\rho \mu}$ provided by the $a$ dependence on the action (14) is written as

$$
t_{\rho \mu}(a, \theta)=x_{\rho \mu}(a, \theta)-\frac{a}{2 \theta^{2}} g_{\rho \mu} \partial_{\sigma}^{-1} \varphi \star \partial_{\sigma}^{-1} \varphi .
$$

We turn to consider this quantity as the regularization contribution of the EMT for the scalar $\varphi_{\star}^{4}$ theory due to the presence of a term allowing renormalization of the action (14), i.e., $\partial_{\mu}^{-1} \varphi \star \partial_{\mu}^{-1} \varphi$. 


\section{The EMT for the GMRT model in the generalized Moyal space}

This section is devoted to the computation of the EMT of the generalized type GMRT model. Before defining this model we give some definitions and identities satisfied by the star product (3). These will be used to calculate the $\varphi$ and the $\varphi^{a}$ variation of the functional action (for more explanation see [16]). Expanding the generalized $\star$-product (3) as follows,

$$
f \star g \equiv e^{\Delta}(f, g)=\sum_{n=0}^{\infty} \frac{\Delta^{n}}{n !}(f, g), \quad \Delta(f, g)=\frac{i}{2} \theta^{a b}\left(X_{a} f\right)\left(X_{b} g\right),
$$

allows us to define the four operators

$$
\begin{aligned}
& T(\Delta)=\frac{e^{\Delta}-1}{\Delta}, \quad S(\Delta)=\frac{\sinh (\Delta)}{\Delta} \\
& R(\Delta)=\frac{\cosh (\Delta)-1}{\Delta} \quad \text { and } \quad \tilde{X}^{a}=\frac{i}{2} \theta^{a b} X_{b}
\end{aligned}
$$

such that the following identities hold:

$$
\begin{aligned}
f \star g & =f g+X_{a} T(\Delta)\left(f, \widetilde{X}^{a} g\right), \\
f \star g-g \star f & =[f, g]_{\star}=2 X_{a} S(\Delta)\left(f, \widetilde{X}^{a} g\right), \\
f \star g+g \star f & =\{f, g\}_{\star}=2 f g+2 X_{a} R(\Delta)\left(f, \widetilde{X}^{a} g\right) .
\end{aligned}
$$

$S(\Delta)(\cdot, \tilde{X} \cdot)$ is a bilinear antisymmetric operator, such that

$$
T(\Delta)\left(f, \widetilde{X}^{a} g\right)-T(\Delta)\left(g, \tilde{X}^{a} f\right)=2 S(\Delta)\left(f, \widetilde{X}^{a} g\right) .
$$

The integral of the form $\int \mathrm{d}^{d} x(f \star g)$ is not cyclic; even with suitable boundary conditions at infinity, i.e.

$$
\int \mathrm{d}^{d} x(f \star g) \neq \int \mathrm{d}^{d} x(g \star f)
$$

Using now the measure $e d^{d} x$, where $e=\operatorname{det}\left(e_{\mu}^{a}\right)$, a cyclic integral can be defined so that, up to boundary terms,

$$
\int e \mathrm{~d}^{d} x(f \star g)=\int e \mathrm{~d}^{d} x(f g)=\int e \mathrm{~d}^{d} x(g \star f) .
$$

From now the peculiar Euler Lagrange equations of motion can be readily derived by direct application of the variation principle and the use of formulas of derivatives and variations given in [16] by

$$
\delta_{\varphi^{c}} e=e X_{a}\left(\delta \varphi^{a}\right), \quad \delta_{\varphi^{c}} e^{-1}=-e^{-1} X_{a}\left(\delta \varphi^{a}\right), \quad e X_{a}(f)=\partial_{\mu}\left(e e_{a}^{\mu} f\right) .
$$

To compute $\delta_{\varphi^{c}}$ variations, consider the functions $f$ and $g$, which do not depend on $\varphi^{c}$. It turns out that the following identity is useful:

$$
\delta_{\varphi^{c}}(f \star g)=-\left(\delta \varphi^{c} X_{c} f\right) \star g-f \star\left(\delta \varphi^{c} X_{c} g\right)+\delta \varphi^{c} X_{c}(f \star g) .
$$

In view of all these considerations, let us suppose now, and in the following, that the field theory is defined by the so-called generalized GMRT model, which is described by the functional action

$$
\begin{aligned}
S_{\star}[\varphi]= & \int e \mathrm{~d}^{d} x\left\{\frac{1}{2} \partial_{\mu} \varphi \star \partial^{\mu} \varphi+\frac{a}{2 \theta^{2}} \partial_{\mu}^{-1} \varphi \star \partial_{\mu}^{-1} \varphi+\frac{m^{2}}{2} \varphi \star \varphi+\frac{\lambda}{4 !} \varphi \star \varphi \star \varphi \star \varphi\right. \\
& \left.+\frac{1}{2} \partial_{\mu} \varphi_{c} \star \partial^{\mu} \varphi^{c}+\frac{a}{2 \theta^{2}} \partial_{\mu}^{-1} \varphi_{c} \star \partial_{\mu}^{-1} \varphi^{c}\right\} \star e^{-1} \\
= & \int e \mathrm{~d}^{d} x\left(\mathcal{L}_{\star} \star e^{-1}\right) .
\end{aligned}
$$

Using expression (36), the action (39) can also be written as $S_{\star}[\varphi]=\int \mathrm{d}^{d} x \mathcal{L}_{\star}$, and then we can easily show that (39) is invariant under spacetime translations. The application of the Noether method to this action which admit continuous symmetrie yields locally conserved EMT, such that the following result holds. 
Proposition 2. The symmetric locally conserved EMT derived from the translation invariance of the action (39) is given by the following relation:

$$
\begin{aligned}
\mathcal{T}_{\nu \sigma}^{s}= & \frac{e}{4}\left[\left(\partial_{\nu} \varphi\right)\left\{\partial_{\sigma} \varphi, e^{-1}\right\}_{\star}+\left(\partial_{\nu} \varphi_{c}\right)\left\{\partial_{\sigma} \varphi^{c}, e^{-1}\right\}_{\star}+\left(\partial_{\sigma} \varphi\right)\left\{\partial_{\nu} \varphi, e^{-1}\right\}_{\star}+\left(\partial_{\sigma} \varphi_{c}\right)\left\{\partial_{\nu} \varphi^{c}, e^{-1}\right\}_{\star}\right] \\
& +\frac{a}{4 \theta^{2}}\left[\partial_{\sigma}^{-1}\left(\partial_{\nu} \varphi\right) \partial_{\sigma}^{-1}\left(e\left\{\partial_{\sigma}^{-1} \varphi, e^{-1}\right\}_{\star}\right)+\partial_{\sigma}^{-1}\left(\partial_{\nu} \varphi_{c}\right) \partial_{\sigma}^{-1}\left(e\left\{\partial_{\sigma}^{-1} \varphi^{c}, e^{-1}\right\}_{\star}\right)\right. \\
& \left.+\partial_{\nu}^{-1}\left(\partial_{\sigma} \varphi\right) \partial_{\nu}^{-1}\left(e\left\{\partial_{\nu}^{-1} \varphi, e^{-1}\right\}_{\star}\right)+\partial_{\nu}^{-1}\left(\partial_{\sigma} \varphi_{c}\right) \partial_{\nu}^{-1}\left(e\left\{\partial_{\nu}^{-1} \varphi^{c}, e^{-1}\right\}_{\star}\right)\right] \\
& -\frac{e}{2}\left\{g_{\rho \sigma} e_{b}^{\rho}\left[\mathcal{L}_{\star} \star\left(e^{-1} \partial_{\nu} \varphi^{b}\right)+T(\Delta)\left(X_{c} \mathcal{L}_{\star}, \widetilde{X}^{b}\left(e^{-1} \partial_{\nu} \varphi^{c}\right)\right)\right]\right. \\
& \left.+g_{\rho \nu} e_{b}^{\rho}\left[\mathcal{L}_{\star} \star\left(e^{-1} \partial_{\sigma} \varphi^{b}\right)+T(\Delta)\left(X_{c} \mathcal{L}_{\star}, \widetilde{X}^{b}\left(e^{-1} \partial_{\sigma} \varphi^{c}\right)\right)\right]\right\} .
\end{aligned}
$$

As mentioned in the last section, the triple index appearing in (40) is not summed. Using the fact that $\partial_{\mu}^{-1} \partial_{\rho} \varphi=$ $\delta_{\mu \rho} \varphi$, the tensor $\widetilde{x}_{\nu \sigma}$ corresponding to $x_{\nu \sigma}$ given in (27) by replacing the Moyal product (1) by (3) is

$$
\begin{aligned}
\widetilde{x}_{\nu \sigma}(a, \theta)= & \frac{a}{4 \theta^{2}}\left[\varphi \int \mathrm{d}^{\sigma} x\left(e\left\{\int \mathrm{d}^{\sigma} x \varphi, e^{-1}\right\}_{\star}\right)+\varphi_{c} \int \mathrm{d}^{\sigma} x\left(e\left\{\int \mathrm{d}^{\sigma} x \varphi^{c}, e^{-1}\right\}_{\star}\right)\right. \\
& \left.+\varphi \int \mathrm{d}^{\nu} x\left(e\left\{\int \mathrm{d}^{\nu} x \varphi, e^{-1}\right\}_{\star}\right)+\varphi_{c} \int \mathrm{d}^{\nu} x\left(e\left\{\int \mathrm{d}^{\nu} x \varphi^{c}, e^{-1}\right\}_{\star}\right)\right] .
\end{aligned}
$$

Then the tensor $\widetilde{t}_{\nu \sigma}$ providing from the $a$ dependence on the action (39) is written as

$$
\begin{aligned}
\widetilde{t}_{\nu \sigma}(a, \theta)= & \widetilde{x}_{\nu \sigma}(a, \theta)-\frac{e}{2}\left\{g_{\rho \sigma} e_{b}^{\rho}\left[\mathcal{L}_{\star}^{(a)} \star\left(e^{-1} \partial_{\nu} \varphi^{b}\right)+T(\Delta)\left(X_{c} \mathcal{L}_{\star}^{(a)}, \widetilde{X}^{b}\left(e^{-1} \partial_{\nu} \varphi^{c}\right)\right)\right]\right. \\
& \left.+g_{\rho \nu} e_{b}^{\rho}\left[\mathcal{L}_{\star}^{(a)} \star\left(e^{-1} \partial_{\sigma} \varphi^{b}\right)+T(\Delta)\left(X_{c} \mathcal{L}_{\star}^{(a)}, \widetilde{X}^{b}\left(e^{-1} \partial_{\sigma} \varphi^{c}\right)\right)\right]\right\},
\end{aligned}
$$

where

$$
\mathcal{L}_{\star}^{(a)}=\frac{a}{2 \theta^{2}}\left(\partial_{\mu}^{-1} \varphi \star \partial_{\mu}^{-1} \varphi+\partial_{\mu}^{-1} \varphi_{c} \star \partial_{\mu}^{-1} \varphi^{c}\right) .
$$

The rest of this paper is devoted to the prove of proposition 2. Let us recall that the case where $a=0$ in (39) is reduced to the well-known scalar field theory in the literature (see [16] for more details). Then we will focus our attention on the $\varphi$ and $\varphi^{c}$ variation of the quantity

$$
S_{\partial}=\frac{a}{2 \theta^{2}} \int e \mathrm{~d}^{d} x\left[\partial_{\mu}^{-1} \varphi \star \partial_{\mu}^{-1} \varphi+\partial_{\mu}^{-1} \varphi_{c} \star \partial_{\mu}^{-1} \varphi^{c}\right] \star e^{-1} .
$$

See the appendix for more details.

The $\varphi$ variation of action (39) gives the EL equations of motion of the $\varphi$ field as

$$
E_{\varphi}=-\frac{1}{2} \partial_{\mu}\left(e\left\{\partial^{\mu} \varphi, e^{-1}\right\}_{\star}\right)-\frac{a}{2 \theta^{2}} \partial_{\mu}^{-1}\left(e\left\{\partial_{\mu}^{-1} \varphi, e^{-1}\right\}_{\star}\right)+\frac{m^{2}}{2} e\left\{\varphi, e^{-1}\right\}_{\star}+\frac{\lambda}{4 !} e\left\{\varphi \star \varphi,\left\{\varphi, e^{-1}\right\}_{\star}\right\}_{\star}=0,
$$

which is reduced to (18) in the limit where $X_{a} \rightarrow \partial_{a}$. Hence, the corresponding current is

$$
\begin{aligned}
\mathcal{K}^{\sigma}= & \frac{a}{2 \theta^{2}}\left[\left(\partial_{\sigma}^{-1} \delta \varphi\right) \partial_{\sigma}^{-1}\left(e\left\{\partial_{\sigma}^{-1} \varphi, e^{-1}\right\}_{\star}\right)+e e_{b}^{\sigma} T(\Delta)\left(\partial_{\mu}^{-1} \delta \varphi, \widetilde{X}^{b}\left(\left\{\partial_{\mu}^{-1} \varphi, e^{-1}\right\}_{\star}\right)\right)\right. \\
& \left.+2 e e_{b}^{\sigma} S(\Delta)\left(\partial_{\mu}^{-1} \varphi, \widetilde{X}^{b}\left(\partial_{\mu}^{-1} \delta \varphi \star e^{-1}\right)\right)\right]+\frac{e \delta \varphi}{2} \cdot\left\{\partial^{\sigma} \varphi, e^{-1}\right\}_{\star} \\
& +e e_{b}^{\sigma}\left[T(\Delta)\left(\delta \partial_{\mu} \varphi, \frac{\widetilde{X}^{b}}{2}\left\{\partial^{\mu} \varphi, e^{-1}\right\}_{\star}\right)+S(\Delta)\left(\partial_{\mu} \varphi, \widetilde{X}^{b}\left(\partial^{\mu} \delta \varphi \star e^{-1}\right)\right)\right] \\
& +e e_{b}^{\sigma}\left[\frac{m^{2}}{2} T(\Delta)\left(\delta \varphi, \widetilde{X}^{b}\left\{\varphi, e^{-1}\right\}_{\star}\right)+m^{2} S(\Delta)\left(\varphi, \widetilde{X}^{b}\left(\delta \varphi \star e^{-1}\right)\right)\right] \\
& +e e_{b}^{\sigma}\left[\frac{\lambda}{4 !} T(\Delta)\left(\delta \varphi, \widetilde{X}^{b}\left\{\varphi \star \varphi,\left\{\varphi, e^{-1}\right\}_{\star}\right\}_{\star}\right)+\frac{\lambda}{12} S(\Delta)\left(\varphi, \widetilde{X}^{b}\left(\delta \varphi \star \varphi \star \varphi \star e^{-1}\right)\right)\right. \\
& \left.+\frac{\lambda}{12} S(\Delta)\left(\varphi \star \varphi, \widetilde{X}^{b}\left(\delta \varphi \star \varphi \star e^{-1}\right)\right)+\frac{\lambda}{12} S(\Delta)\left(\varphi \star \varphi \star \varphi, \widetilde{X}^{b}\left(\delta \varphi \star e^{-1}\right)\right)\right],
\end{aligned}
$$


such that

$$
\delta_{\varphi} S_{\star}=\int \mathrm{d}^{d} x\left[\delta \varphi E^{\varphi}+\partial_{\sigma} \mathcal{K}^{\sigma}\right]
$$

In the other hand the $\varphi_{c}$ variation of (39) gives the EL-equations of motion

$$
\begin{aligned}
E^{\varphi \varphi^{c}}= & E_{\partial}^{\varphi^{c}}-X_{c} \varphi \mathcal{E}_{\varphi}+X_{c} \mathcal{L}_{\star}^{\Omega}-\frac{1}{2} X_{c} \varphi \partial_{\mu}\left(e\left\{\partial^{\mu} \varphi, e^{-1}\right\}_{\star}\right)-e \frac{\Omega^{2}}{2} \varphi X_{c} \tilde{x} \cdot\left\{\tilde{x} \varphi, e^{-1}\right\}_{\star} \\
& -\frac{e}{2} X_{c} \partial_{\mu} \varphi \cdot\left\{\partial^{\mu} \varphi, e^{-1}\right\}_{\star}-\frac{e}{2} X_{c} \partial_{\mu} \varphi_{a} \cdot\left\{\partial^{\mu} \varphi^{a}, e^{-1}\right\}_{\star}-\partial_{\mu}\left(\frac{e}{2}\left\{\partial^{\mu} \varphi_{c}, e^{-1}\right\}_{\star}\right)=0
\end{aligned}
$$

and the corresponding current

$$
\begin{aligned}
\mathcal{J}^{\sigma}= & \mathcal{J}_{\partial}^{\sigma}+\mathcal{K}^{\sigma}\left(\delta \varphi \rightarrow-\delta \varphi^{c} X_{c} \varphi\right)+\frac{e \delta \varphi^{c}}{2} X_{c} \varphi \cdot\left\{\partial^{\sigma} \varphi, e^{-1}\right\}_{\star}+\frac{e \delta \varphi^{c}}{2} \cdot\left\{\partial^{\sigma} \varphi_{c}, e^{-1}\right\}_{\star} \\
& +e e_{b}^{\sigma}\left\{-\mathcal{L}_{\star} \star\left(\delta \varphi^{b} e^{-1}\right)+\delta \varphi^{b}\left(\mathcal{L}_{\star} \star e^{-1}\right)+T(\Delta)\left(X_{c}\left(\mathcal{L}_{\star}\right), \widetilde{X}^{b}\left(\delta \varphi^{c} e^{-1}\right)\right)\right. \\
& \left.+\frac{1}{2} T(\Delta)\left(\partial_{\mu}\left(\delta \varphi^{c} e_{c}^{\rho}\right) \partial_{\rho} \varphi, \widetilde{X}^{b}\left\{\partial^{\mu} \varphi, e^{-1}\right\}_{\star}\right)+S(\Delta)\left(\partial_{\mu} \varphi, \widetilde{X}^{b}\left(\left(\partial_{\mu}\left(\delta \varphi^{c} e_{c}^{\rho}\right) \partial_{\rho} \varphi\right) \star e^{-1}\right)\right)\right\} \\
& +\frac{1}{2} e e_{b}^{\sigma}\left\{-T(\Delta)\left(\delta \varphi^{c} X_{c} \partial_{\mu} \varphi_{a}, \widetilde{X}^{b}\left\{\partial^{\mu} \varphi^{a}, e^{-1}\right\}_{\star}\right)-2 S(\Delta)\left(\partial^{\mu} \varphi_{a}, \widetilde{X}^{b}\left(\left(\delta \varphi^{c} X_{c} \partial_{\mu} \varphi^{a}\right) \star e^{-1}\right)\right)\right. \\
& \left.+2 S(\Delta)\left(\partial_{\mu} \varphi_{a}, \widetilde{X}^{b}\left(\partial^{\mu} \delta \varphi^{a} \star e^{-1}\right)\right)+T(\Delta)\left(\partial_{\mu} \delta \varphi_{a}, \widetilde{X}^{b}\left\{\partial^{\mu} \varphi^{a}, e^{-1}\right\}_{\star}\right)\right\},
\end{aligned}
$$

such that

$$
\delta_{\varphi^{c}} \mathcal{S}_{\star}=\int \mathrm{d}^{d} x\left(\delta \varphi^{c} E^{\varphi \varphi^{c}}+\partial_{\sigma} \mathcal{J}^{\sigma}\right) .
$$

Now using the results in the previous paragraph where we studied the general properties of the total variation of the Lagrangian, we discuss the translation invariant symmetry of the model and compute the conserved current, namely, the EMT. In general, a symmetry of the action involves a certain change of variables. Performing a functional variation of the fields and a coordinates transformations

$$
\varphi^{\prime}(x)=\varphi(x)+\delta \varphi(x), \quad \varphi^{\prime c}(x)=\varphi^{c}(x)+\delta \varphi^{c}(x), \quad x^{\prime \mu}=x^{\mu}+a^{\mu},
$$

and by using the identity $\mathrm{d}^{D} x^{\prime}=\left[1+\partial_{\mu} a^{\mu}+\mathcal{O}\left(a^{2}\right)\right] \mathrm{d}^{D} x$, leads to the following variation of the action, to first order in $\delta \varphi(x), \delta \varphi^{c}(x)$ and $a^{\mu}$ :

$$
\begin{aligned}
\delta \mathcal{S}_{\star} & =\int e \mathrm{~d}^{d} x\left\{\left|\frac{\partial x^{\prime}}{\partial x}\right| \star\left(\mathcal{L}^{\prime} \star \star e^{-1}\right)\right\}-\int e \mathrm{~d}^{d} x\left(\mathcal{L}_{\star} \star e^{-1}\right) \\
& =\int \mathrm{d}^{d} x\left\{\delta_{\varphi}\left(\left(\mathcal{L}_{\star} \star e^{-1}\right) e\right)+\delta_{\varphi^{c}}\left(\left(\mathcal{L}_{\star} \star e^{-1}\right) e\right)+a^{\mu} \star \partial_{\mu}\left[\left(\mathcal{L}_{\star} \star e^{-1}\right) e\right]+\partial_{\mu} a^{\mu} \star\left(\mathcal{L}_{\star} \star e^{-1}\right) e\right\}
\end{aligned}
$$

Now by integrating on a submanifold $M \subset \mathbb{R}^{D}$ with nonvanishing fields at the boundary (so that the total derivative terms do not disappear), we get

$$
\delta \mathcal{S}_{\star}=\int_{M} \mathrm{~d}^{d} x \partial_{\sigma}\left[\mathcal{K}^{\sigma}+\mathcal{J}^{\sigma}+a^{\sigma} \star\left(\left(\mathcal{L}_{\star}^{\Omega} \star e^{-1}\right) e\right)\right]
$$

coupled to the transformations $\delta \varphi=-a^{\nu} \partial_{\nu} \varphi, \delta \varphi^{c}=-a^{\nu} \partial_{\nu} \varphi^{c}, a^{\nu}=$ constant, that we substitute into (52) and, taking into account the identities $\delta \varphi^{c} X_{c} \partial_{\mu} \varphi=\partial_{\mu}\left(\delta \varphi^{c} X_{c} \varphi\right)-\partial_{\mu}\left(\delta \varphi^{c} e_{c}^{\rho}\right) \partial_{\rho} \varphi$, such that $\delta \varphi^{c} X_{c} \partial_{\mu} \varphi=\partial_{\mu} \delta \varphi=-a^{\nu} \partial_{\nu} \partial_{\mu} \varphi$, and the fact that $e_{\nu}^{a}=\partial_{\nu} \varphi^{a}$, we come to the relation

$$
0=\delta \mathcal{S}_{\star}=-a^{\nu} \int_{M} \mathrm{~d}^{d} x \partial_{\sigma} \mathcal{T}_{\nu}^{\sigma}
$$

where the EMT takes the form

$$
\begin{aligned}
\mathcal{T}_{\nu}^{\sigma}= & \frac{e}{2}\left[\left(\partial_{\nu} \varphi\right)\left\{\partial^{\sigma} \varphi, e^{-1}\right\}_{\star}+\left(\partial_{\nu} \varphi_{c}\right)\left\{\partial^{\sigma} \varphi^{c}, e^{-1}\right\}_{\star}\right] \\
& +\frac{a}{2 \theta^{2}}\left[\partial_{\sigma}^{-1}\left(\partial_{\nu} \varphi\right) \partial_{\sigma}^{-1}\left(e\left\{\partial_{\sigma}^{-1} \varphi, e^{-1}\right\}_{\star}\right)+\partial_{\sigma}^{-1}\left(\partial_{\nu} \varphi_{c}\right) \partial_{\sigma}^{-1}\left(e\left\{\partial_{\sigma}^{-1} \varphi^{c}, e^{-1}\right\}_{\star}\right)\right] \\
& -e e_{b}^{\sigma}\left[\mathcal{L}_{\star} \star\left(e^{-1} \partial_{\nu} \varphi^{b}\right)+T(\Delta)\left(X_{c} \mathcal{L}_{\star}, \tilde{X}^{b}\left(e^{-1} \partial_{\nu} \varphi^{c}\right)\right)\right] .
\end{aligned}
$$


This tensor is not symmetric and nonlocally conserved. Note that to recover the EMT given in (19) we write $T_{\nu \rho}=$ $g_{\sigma \rho} \mathcal{T}_{\nu}^{\sigma}$ and take the limit $e_{a}^{\mu} \rightarrow \delta_{a}^{\mu}$. Expression (54) can be symmetrized as

$$
\begin{aligned}
\mathcal{T}_{\nu \sigma}^{s}= & \frac{e}{4}\left[\left(\partial_{\nu} \varphi\right)\left\{\partial_{\sigma} \varphi, e^{-1}\right\}_{\star}+\left(\partial_{\nu} \varphi_{c}\right)\left\{\partial_{\sigma} \varphi^{c}, e^{-1}\right\}_{\star}+\left(\partial_{\sigma} \varphi\right)\left\{\partial_{\nu} \varphi, e^{-1}\right\}_{\star}+\left(\partial_{\sigma} \varphi_{c}\right)\left\{\partial_{\nu} \varphi^{c}, e^{-1}\right\}_{\star}\right] \\
& +\frac{a}{4 \theta^{2}}\left[\partial_{\sigma}^{-1}\left(\partial_{\nu} \varphi\right) \partial_{\sigma}^{-1}\left(e\left\{\partial_{\sigma}^{-1} \varphi, e^{-1}\right\}_{\star}\right)+\partial_{\sigma}^{-1}\left(\partial_{\nu} \varphi_{c}\right) \partial_{\sigma}^{-1}\left(e\left\{\partial_{\sigma}^{-1} \varphi^{c}, e^{-1}\right\}_{\star}\right)\right. \\
& \left.+\partial_{\nu}^{-1}\left(\partial_{\sigma} \varphi\right) \partial_{\nu}^{-1}\left(e\left\{\partial_{\nu}^{-1} \varphi, e^{-1}\right\}_{\star}\right)+\partial_{\nu}^{-1}\left(\partial_{\sigma} \varphi_{c}\right) \partial_{\nu}^{-1}\left(e\left\{\partial_{\nu}^{-1} \varphi^{c}, e^{-1}\right\}_{\star}\right)\right] \\
& -\frac{e}{2}\left\{g_{\rho \sigma} e_{b}^{\rho}\left[\mathcal{L}_{\star} \star\left(e^{-1} \partial_{\nu} \varphi^{b}\right)+T(\Delta)\left(X_{c} \mathcal{L}_{\star}, \widetilde{X}^{b}\left(e^{-1} \partial_{\nu} \varphi^{c}\right)\right)\right]\right. \\
& \left.+g_{\rho \nu} e_{b}^{\rho}\left[\mathcal{L}_{\star} \star\left(e^{-1} \partial_{\sigma} \varphi^{b}\right)+T(\Delta)\left(X_{c} \mathcal{L}_{\star}, \widetilde{X}^{b}\left(e^{-1} \partial_{\sigma} \varphi^{c}\right)\right)\right]\right\} .
\end{aligned}
$$

Now we can regularize the EMT (54). Due to the very complex form of expression in the general case, we focus our attention on the case where the coordinates base $e_{a}^{\mu}(x)$ is to be $e_{a}^{\mu}=\delta_{a}^{\mu}+\omega_{a b}^{\mu} x^{b}$, such that the tensor $\left(\omega_{a b}^{\mu}\right)$ is symmetric between the index $a$ and $b$, i.e. $\omega_{a b}^{\mu}=\omega_{b a}^{\mu}$. The commutation relation between the vectors fields $X_{a}$ is

$$
\left[X_{a}, X_{b}\right]=\left(\omega_{b a}^{\mu}-\omega_{a b}^{\mu}\right) \partial_{\mu}=0
$$

and therefore the dynamical star product is associative. We adequately choose the elements of the matrix $\left(\omega_{a b}^{\mu}\right)$, such that the matrix representation of $\left(e_{a}^{\mu}\right)$ is given in dimension $d=4$ by

$$
(e)_{a}^{\mu}=\left(\begin{array}{cccc}
1+\omega_{11}^{1} x^{1}+\omega_{12}^{1} x^{2} & \omega_{11}^{2} x^{1}+\omega_{12}^{2} x^{2} & 0 & 0 \\
\omega_{12}^{1} x^{1}+\omega_{22}^{1} x^{2} & 1+\omega_{12}^{2} x^{1}+\omega_{22}^{2} x^{2} & 0 & 0 \\
0 & 0 & 1+\omega_{33}^{3} x^{3}+\omega_{34}^{3} x^{4} & \omega_{33}^{4} x^{3}+\omega_{34}^{4} x^{4} \\
0 & 0 & \omega_{34}^{3} x^{3}+\omega_{44}^{3} x^{4} & 1+\omega_{34}^{4} x^{3}+\omega_{44}^{4} x^{4}
\end{array}\right) .
$$

Then, the determinants $e^{-1}$ and the inverse $e$ become

$$
e^{-1}=1+\omega_{\mu} x^{\mu}, \quad e=1-\omega_{\mu} x^{\mu},
$$

where the components of the vector $\omega_{\mu}$ are

$$
\omega_{1}=\omega_{11}^{1}+\omega_{12}^{2}, \quad \omega_{2}=\omega_{22}^{2}+\omega_{12}^{1}, \quad \omega_{3}=\omega_{33}^{3}+\omega_{34}^{4}, \quad \omega_{4}=\omega_{44}^{4}+\omega_{34}^{3} .
$$

The noncommutative tensor takes the form $\theta^{\mu \nu}(x)=\theta e^{-1} J^{\mu \nu}$, where $(J)$ stands for the symplectic matrix in four dimensions. Besides, the inverse matrix $e_{\mu}^{a}$ can be written as $e_{\mu}^{a}=\delta_{\mu}^{a}+\omega_{\mu}^{a b} x_{b}$, where $\omega_{\mu}^{a b}=-\omega_{a b}^{\mu}$, and the solution of the field equation $e_{\mu}^{a}=\partial_{\mu} \phi^{a}$ is given by

$$
\phi^{a}=x^{a}+\frac{1}{2} \omega_{\mu}^{a b} x_{b} x^{\mu}
$$

Using all these considerations, after little algebraic computation, we come to the relation

$$
\begin{aligned}
\partial^{\nu} \mathcal{T}_{\nu \sigma}^{s} & =\frac{2 e \lambda}{4 !} X_{a} S(\Delta)\left(\left[\partial_{\sigma} \varphi, \varphi\right]_{\star}, \tilde{X}^{a}\left(\varphi \star \varphi \star e^{-1}\right)\right) \\
& =\frac{2 \lambda}{4 !} \partial_{\gamma}\left(e e_{a}^{\gamma} S(\Delta)\left(\left[\partial_{\sigma} \varphi, \varphi\right]_{\star}, \tilde{X}^{a}\left(\varphi \star \varphi \star e^{-1}\right)\right)\right),
\end{aligned}
$$

where the following identities are used

$$
\begin{aligned}
& \left\{\partial_{\sigma} \varphi, e^{-1}\right\}_{\star}=2 e^{-1} \partial_{\sigma} \varphi, \\
& e^{-1} \partial_{\sigma} \varphi^{c}=\delta_{\sigma}^{c}+\delta_{\sigma}^{c} \omega_{\mu} x^{\mu}+\omega_{\sigma}^{c d} x_{d}, \\
& T(\Delta)\left(X_{c} \mathcal{L}_{\star}, \widetilde{X}^{b}\left(e^{-1} \partial_{\sigma} \varphi^{c}\right)\right)=0 .
\end{aligned}
$$

As the ordinary Moyal plane, the EMT defined on the dynamical Moyal space can be regularized. We get the symmetric tensor

$$
\mathcal{T}_{\nu \sigma}^{s, r}=\mathcal{T}_{\nu \sigma}^{s}-\frac{2 \lambda}{4 !} g_{\gamma \nu}\left(e e_{a}^{\gamma} S(\Delta)\left(\left[\partial_{\sigma} \varphi, \varphi\right]_{\star}, \widetilde{X}^{a}\left(\varphi \star \varphi \star e^{-1}\right)\right)\right)-\frac{2 \lambda}{4 !} g_{\gamma \sigma}\left(e e_{a}^{\gamma} S(\Delta)\left(\left[\partial_{\nu} \varphi, \varphi\right]_{\star}, \widetilde{X}^{a}\left(\varphi \star \varphi \star e^{-1}\right)\right)\right) .
$$

By incorporating noncommutativity in the coordinates, the gravitation interaction can be taken into account in QFTs. However, the computation of the EMTs is based around a prejudice for writing the Einstein field equations as $\mathcal{G}_{\nu \sigma}=\kappa \mathcal{T}_{\nu \sigma}^{s, r}$ with gravity on the left and matter on the right. Due to the fact that $\partial^{\nu} \mathcal{G}_{\nu \sigma}=0$, we need improve the EMT such that $\partial^{\nu} \mathcal{T}_{\nu \sigma}^{s, r}=0$. As in $[29,30,37]$ we have explicitly shown that the standard local conservation law of the EMT is always modified due to noncommutative effects and that tensor can always be redefined so as to be conserved. 


\section{Conclusion and remarks}

In conclusion, we summarize our results. We have developed the variation techniques for the determination of the EL equations of motion of a Lagrangian that depends on $\partial_{\mu}^{-1} \varphi$. We have computed the EMT for the GMRT model, in ordinary and dynamical Moyal spaces. The Wilson regularization procedure is also given to improve the corresponding tensors.

Let us remark the following.

i) The invariance of the action (14) under spacetime translation involves the locally conserved EMT, which needs not be symmetric and in a massless theory, it needs not be traceless either. The Lorentz symmetry in noncommutative theory is broken for $D>2$, since the constant skewsymmetric tensor $\theta^{\mu \nu}$ is not a Lorentz invariant tensor. As is explaned in [16], one way to restore this symmetry is to generalized the Moyal product defined by a set of a commuting vector field $X_{a}=e_{a}^{\mu}(x) \partial_{\mu}$. Therefore the EMT given with the translation invariant renormalizable action (the GMRT model) in the generalized Moyal space seems to be Lorentz invariant tensor.

ii) Introducing $x$ dependence in the deformation matrix $\left(\theta^{\mu \nu}\right)$ of the star product leads to the definition of nontrivial background metric. Then the EMT associated to translation invariant field theory may provided from the core of the Einstein equation, when we assume that gravity can be incorporated in the noncommutativity. Also, the EMT given in (40) can be regularized without choosing the tetrad as $e_{a}^{\mu}=\delta_{a}^{\mu}+\omega_{a b}^{\mu} x^{b}$. In the general case of the tetrad $e_{a}^{\mu}(x)$, the same computation can be made easily, thanks to the example proposed in this paper.

Open access funding provided by Max Planck Society. The authors thank the referee for careful reading and comments, which allowed to improve this manuscript. The work of EB is partially supported by the Abdus Salam International Centre for Theoretical Physics (ICTP, Trieste, Italy) through the Office of External Activities (OEA)-Prj-15. The ICMPA is in partnership with the Daniel Lagolnitzer Foundation (DIF), France. The research of DOS at Max-Planck Institute is supported by the Alexander von Humboldt foundation.

\section{Appendix A. Formal variation principle of a nonlocal action $\mathcal{L}_{\star}$}

In this section we derive the variation principle in a formal way, which allows us to compute the EMT (13). The nonlocal Lagrangian we consider here is of the form $\mathcal{L}_{\star}\left(\varphi, \partial_{\mu} \varphi, \partial_{\mu}^{-1} \varphi\right)$. As mentioned in sect. 2, the nonlocality of $\mathcal{L}_{\star}$ comes not only from the star product, but also from the inverse derivative of the field $\varphi$ denoted by $\partial^{-1} \varphi$. There are two ways for investigating this Lagrangian. The first is to expand the star product and get a quantity of the form

$$
\mathcal{L}_{\star}\left(\varphi, \partial_{\mu} \varphi, \partial_{\mu}^{-1} \varphi\right)=\mathcal{L}\left(\varphi, \partial_{\mu} \varphi, \cdots, \infty, \partial^{-1} \varphi, \theta\right) .
$$

The right-hand side of this expression can be treated using the generalization of the Ostrogradski calculus for $n$ derivative Lagragian, see [38-41] for more explanations about this method. On the other hand, the left-hand side may be treated as nonlocal noncommutative Lagrangian, and then the computation of the EMT is similar to what follows in [29-37], apart from the fact that the inverse derivative needs to be considered carefully. Let $S_{\star}[\varphi]=$ $\int \mathrm{d}^{d} x \mathcal{L}_{\star}\left(\varphi, \partial_{\mu} \varphi, \Psi\right)$ be the nonlocal action coming from the nonlocal Lagrangian (A.1), in which the quantity $\partial^{-1} \varphi$ is considered as a new field denoted by $\Psi$; we get the following variation:

$$
\delta S_{\star}[\varphi]=\int \mathrm{d}^{d} x\left(\frac{\partial \mathcal{L}_{\star}}{\partial \varphi} \star \delta \varphi+\frac{\partial \mathcal{L}_{\star}}{\partial \partial_{\mu} \varphi} \star \delta \partial_{\mu} \varphi+\frac{\partial \mathcal{L}_{\star}}{\partial \Psi} \star \delta \Psi\right) .
$$

Now, by considering the following identities,

$$
\partial_{\mu} \partial_{\nu}^{-1} \varphi(x)=\delta_{\mu \nu} \varphi(x), \quad \partial_{\mu}(a \star b)=\left(\partial_{\mu} a\right) \star b+a \star\left(\partial_{\mu} b\right),
$$

we can simply deduce that

$$
\begin{aligned}
\frac{\partial \mathcal{L}_{\star}}{\partial \partial_{\mu} \varphi} \star \partial_{\mu} \delta \varphi & =\partial_{\mu}\left(\frac{\partial \mathcal{L}_{\star}}{\partial \partial_{\mu} \varphi} \star \delta \varphi\right)-\partial_{\mu}\left(\frac{\partial \mathcal{L}_{\star}}{\partial \partial_{\mu} \varphi}\right) \star \delta \varphi \\
\frac{\partial \mathcal{L}_{\star}}{\partial \Psi} \star \delta \Psi & =\partial_{\mu}\left[\partial_{\mu}^{-1}\left(\frac{\partial \mathcal{L}_{\star}}{\partial \Psi}\right) \star \delta \Psi\right]-\partial_{\mu}^{-1}\left(\frac{\partial \mathcal{L}_{\star}}{\partial \Psi}\right) \star \delta \varphi 1_{\mu}
\end{aligned}
$$

where the vector notation $1_{\mu}=\delta_{\mu \mu}$ is used to point out the fact that the Einstein summation holds. Then (A.2) becomes

$$
\delta S_{\star}[\varphi]=\int \mathrm{d}^{d} x\left(E_{\varphi} \delta \varphi+\partial^{\mu} J_{\mu}\right)
$$


where the quantities $E_{\varphi}$ and $J_{\mu}$ are, respectively, given by

$$
\begin{aligned}
E_{\varphi}: & =\frac{\partial \mathcal{L}_{\star}}{\partial \varphi}-\partial_{\mu}\left(\frac{\partial \mathcal{L}_{\star}}{\partial \partial_{\mu} \varphi}\right)-\partial_{\mu}^{-1}\left(\frac{\partial \mathcal{L}_{\star}}{\partial \Psi}\right) 1_{\mu}, \\
J_{\mu}: & =\left(\frac{\partial \mathcal{L}_{\star}}{\partial \partial_{\mu} \varphi} \star \delta \varphi\right)+\partial_{\mu}^{-1}\left(\frac{\partial \mathcal{L}_{\star}}{\partial \Psi}\right) \star \delta \Psi
\end{aligned}
$$

The current $J_{\mu}$ involves the infinite number of derivatives respect to the field $\varphi$ due to the definition of the star product, also the inverse derivative of the form $\partial_{\mu}^{-1} \varphi, \partial_{\mu}^{-2} \varphi$ appears in this quantity. Let $\partial^{\alpha} \varphi \in \mathcal{S}\left(\mathbb{R}^{d}\right), \alpha=\llbracket-2,-1 \rrbracket \cup \mathbb{N}$, where $\mathcal{S}\left(\mathbb{R}^{d}\right)$ is the space of suitable Schwartzian functions. With this condition, the surface term vanishes, i.e. $\int \mathrm{d}^{d} x \partial^{\mu} J_{\mu}=0$, and the EL equation of motion $E_{\varphi}=0$ is well satisfied. In the case of translation invariant action, the coordinates and field are transformed as

$$
x^{\prime \mu}=x^{\mu}+a^{\mu}, \quad d^{d} x^{\prime}=\left[1+\partial_{\mu} a^{\mu}+\mathcal{O}\left(a^{2}\right)\right] d^{d} x, \quad \delta \varphi=-a^{\mu} \partial_{\mu} \varphi,
$$

such that the variation of the action $S_{\star}$ becomes ( $a^{\mu}$ is a constant vector):

$$
\begin{aligned}
\delta S_{\star} & =\int \mathrm{d}^{d} x\left|\frac{\partial x^{\prime}}{\partial x}\right| \star \mathcal{L}_{\star}^{\prime}-\int \mathrm{d}^{d} x \mathcal{L}_{\star} \\
& =\int \mathrm{d}^{d} x\left(\delta_{\varphi} \mathcal{L}_{\star}+a^{\mu} \partial_{\mu} \mathcal{L}_{\star}\right) .
\end{aligned}
$$

Finally, taking into account the fact that $E_{\varphi}=0$, we come to

$$
\begin{aligned}
\delta S_{\star} & =\int \mathrm{d}^{d} x\left[\partial^{\nu} J_{\nu}\left(\delta \varphi=-a_{\mu} \partial^{\mu} \varphi\right)+a^{\mu} g_{\mu \nu} \partial^{\nu} \mathcal{L}_{\star}\right] \\
& =-a^{\mu} \int \mathrm{d}^{d} x \partial^{\nu}\left[\left(\frac{\partial \mathcal{L}_{\star}}{\partial \partial_{\nu} \varphi} \star \partial_{\mu} \varphi\right)+\partial_{\nu}^{-1}\left(\frac{\partial \mathcal{L}_{\star}}{\partial \Psi}\right) \star \partial_{\mu} \Psi-g_{\mu \nu} \mathcal{L}_{\star}\right] \\
& =-a^{\mu} \int \mathrm{d}^{d} x \partial^{\nu}\left[\frac{1}{2}\left\{\frac{\partial \mathcal{L}_{\star}}{\partial \partial_{\nu} \varphi}, \partial_{\mu} \varphi\right\}_{\star}+\frac{1}{2}\left\{\partial_{\nu}^{-1}\left(\frac{\partial \mathcal{L}_{\star}}{\partial \Psi}\right), \partial_{\mu} \Psi\right\}_{\star}-g_{\mu \nu} \mathcal{L}_{\star}\right] \\
& =-a^{\mu} \int \mathrm{d}^{d} x \partial^{\nu} T_{\nu \mu} .
\end{aligned}
$$

\section{Appendix B. Proof of relations (46) and (49)}

In this appendix we give the proof of the variation principle, which leads to the EL equations of motion and the corresponding current and therefore contribute to the proof of relations (46) and (49). Consider the action (43). Recall that the $\varphi_{c}$ field does not depend on $\varphi$. We get

$$
\begin{aligned}
\delta_{\varphi} S_{\partial}= & \frac{a}{2 \theta^{2}} \int e \mathrm{~d}^{d} x\left[\partial_{\mu}^{-1} \delta \varphi \star \partial_{\mu}^{-1} \varphi \star e^{-1}+\partial_{\mu}^{-1} \varphi \star \partial_{\mu}^{-1} \delta \varphi \star e^{-1}\right] \\
= & \frac{a}{2 \theta^{2}} \int e \mathrm{~d}^{d} x\left[\partial_{\mu}^{-1} \delta \varphi \star\left\{\partial_{\mu}^{-1} \varphi, e^{-1}\right\}_{\star}+2 X_{a} S(\Delta)\left(\partial_{\mu}^{-1} \varphi, \widetilde{X}^{a}\left(\partial_{\mu}^{-1} \delta \varphi \star e^{-1}\right)\right)\right] \\
= & \frac{a}{2 \theta^{2}} \int e \mathrm{~d}^{d} x\left[\left(\partial_{\mu}^{-1} \delta \varphi\right)\left\{\partial_{\mu}^{-1} \varphi, e^{-1}\right\}_{\star}+X_{a} T(\Delta)\left(\partial_{\mu}^{-1} \delta \varphi, \widetilde{X}^{a}\left(\left\{\partial_{\mu}^{-1} \varphi, e^{-1}\right\}_{\star}\right)\right)\right. \\
& \left.+2 X_{a} S(\Delta)\left(\partial_{\mu}^{-1} \varphi, \widetilde{X}^{a}\left(\partial_{\mu}^{-1} \delta \varphi \star e^{-1}\right)\right)\right]
\end{aligned}
$$

where we have used the identities

$$
\begin{gathered}
\partial_{\mu}^{-1} \varphi \star \partial_{\mu}^{-1} \delta \varphi \star e^{-1}=\partial_{\mu}^{-1} \delta \varphi \star e^{-1} \star \partial_{\mu}^{-1} \varphi+2 X_{a} S(\Delta)\left(\partial_{\mu}^{-1} \varphi, \tilde{X}^{a}\left(\partial_{\mu}^{-1} \delta \varphi \star e^{-1}\right)\right), \\
\left(\partial_{\mu}^{-1} \delta \varphi\right) \star\left\{\partial_{\mu}^{-1} \varphi, e^{-1}\right\}_{\star}=\left(\partial_{\mu}^{-1} \delta \varphi\right)\left\{\partial_{\mu}^{-1} \varphi, e^{-1}\right\}_{\star}+X_{a} T(\Delta)\left(\partial_{\mu}^{-1} \delta \varphi, \widetilde{X}^{a}\left(\left\{\partial_{\mu}^{-1} \varphi, e^{-1}\right\}_{\star}\right)\right) .
\end{gathered}
$$

Consider the following relation, in which the repetitive indices in the right-hand side are now summed:

$$
e\left(\partial_{\mu}^{-1} \delta \varphi\right)\left\{\partial_{\mu}^{-1} \varphi, e^{-1}\right\}_{\star}=\sum_{\mu} \partial_{\mu}\left[\left(\partial_{\mu}^{-1} \delta \varphi\right) \partial_{\mu}^{-1}\left(e\left\{\partial_{\mu}^{-1} \varphi, e^{-1}\right\}_{\star}\right)\right]-\delta \varphi \partial_{\mu}^{-1}\left(e\left\{\partial_{\mu}^{-1} \varphi, e^{-1}\right\}_{\star}\right) .
$$


By replacing this identity in (B.1), we get the $\varphi$ variation of $S_{\partial}$ as

$$
\delta_{\varphi} S_{\partial}=\int \mathrm{d}^{d} x\left[\delta \varphi E_{\partial}^{\varphi}+\partial_{\sigma} \mathcal{K}_{\partial}^{\sigma}\right]
$$

where $E_{\partial}$ contributes to the EL equations of motion and $\mathcal{K}_{\partial}^{\mu}$ to the current:

$$
\begin{aligned}
E_{\partial}^{\varphi}= & -\frac{a}{2 \theta^{2}} \partial_{\mu}^{-1}\left(e\left\{\partial_{\mu}^{-1} \varphi, e^{-1}\right\}_{\star}\right), \\
\mathcal{K}_{\partial}^{\sigma}= & \frac{a}{2 \theta^{2}}\left[\left(\partial_{\sigma}^{-1} \delta \varphi\right) \partial_{\sigma}^{-1}\left(e\left\{\partial_{\sigma}^{-1} \varphi, e^{-1}\right\}_{\star}\right)+e e_{b}^{\sigma} T(\Delta)\left(\partial_{\mu}^{-1} \delta \varphi, \widetilde{X}^{b}\left(\left\{\partial_{\mu}^{-1} \varphi, e^{-1}\right\}_{\star}\right)\right)\right. \\
& \left.+2 e e_{b}^{\sigma} S(\Delta)\left(\partial_{\mu}^{-1} \varphi, \widetilde{X}^{b}\left(\partial_{\mu}^{-1} \delta \varphi \star e^{-1}\right)\right)\right] .
\end{aligned}
$$

Using the same technical computation to the remain expression of the functional action (39) the EL equations of motion of the $\varphi$ field, i.e. $E^{\varphi}=0$ and the corresponding current $\mathcal{K}^{\sigma}$ given, respectively, in relations (44) and (45) are well satisfied, and then

$$
\delta_{\varphi} S_{\star}=\int \mathrm{d}^{d} x\left[\delta \varphi E^{\varphi}+\partial_{\sigma} \mathcal{K}^{\sigma}\right] .
$$

On the other hand, we are interested in the $\varphi_{c}$ variation of (43). This variation is subdivided into two contributions, namely $A_{\partial}$ and $B_{\partial}$, such that

$$
\begin{aligned}
\delta_{\varphi^{c}} S_{\partial} & =\frac{a}{2 \theta^{2}} \delta_{\varphi^{c}}\left\{\int e \mathrm{~d}^{d} x \partial_{\mu}^{-1} \varphi \star \partial_{\mu}^{-1} \varphi \star e^{-1}\right\}+\frac{a}{2 \theta^{2}} \delta_{\varphi^{c}}\left\{\int e \mathrm{~d}^{d} x \partial_{\mu}^{-1} \varphi_{c} \star \partial_{\mu}^{-1} \varphi^{c} \star e^{-1}\right\} \\
& =A_{\partial}+B_{\partial}
\end{aligned}
$$

where

$$
\begin{aligned}
A_{\partial}= & \frac{a}{2 \theta^{2}} \int \mathrm{d}^{d} x\left(\delta_{\varphi^{c}} e\right)\left(\partial_{\mu}^{-1} \varphi \star \partial_{\mu}^{-1} \varphi \star e^{-1}\right)+\frac{a}{2 \theta^{2}} \int e \mathrm{~d}^{d} x \delta_{\varphi^{c}}\left(\partial_{\mu}^{-1} \varphi \star \partial_{\mu}^{-1} \varphi \star e^{-1}\right) \\
= & \frac{a}{2 \theta^{2}} \int \mathrm{d}^{d} x\left\{\partial_{\sigma}\left(e e_{a}^{\sigma} \delta \varphi^{a} \partial_{\mu}^{-1} \varphi \star \partial_{\mu}^{-1} \varphi \star e^{-1}\right)-e e_{a}^{\sigma} \delta \varphi^{a} \partial_{\sigma}\left(\partial_{\mu}^{-1} \varphi \star \partial_{\mu}^{-1} \varphi \star e^{-1}\right)\right\} \\
& +\frac{a}{2 \theta^{2}} \int e \mathrm{~d}^{d} x\left\{-\delta \varphi^{a} X_{a} \partial_{\mu}^{-1} \varphi\left\{\partial_{\mu}^{-1} \varphi, e^{-1}\right\}_{\star}-X_{b} T(\Delta)\left(\delta \varphi^{a} X_{a} \partial_{\mu}^{-1} \varphi, \widetilde{X}^{b}\left\{\partial_{\mu}^{-1} \varphi, e^{-1}\right\}_{\star}\right)\right. \\
& -2 X_{b} S(\Delta)\left(\partial_{\mu}^{-1} \varphi, \widetilde{X}^{b} \delta \varphi^{a} X_{a} \partial_{\mu}^{-1} \varphi \star e^{-1}\right)-X_{a}\left(\partial_{\mu}^{-1} \varphi \star \partial_{\mu}^{-1} \varphi \star \delta \varphi^{a} e^{-1}\right) \\
& +X_{a}\left(\partial_{\mu}^{-1} \varphi \star \partial_{\mu}^{-1} \varphi\right) \delta \varphi^{a} e^{-1}+X_{b} T(\Delta)\left(X_{a}\left(\partial_{\mu}^{-1} \varphi \star \partial_{\mu}^{-1} \varphi\right), \widetilde{X}^{b} \delta \varphi^{a} e^{-1}\right) \\
& \left.+\delta \varphi^{a} X_{a}\left(\partial_{\mu}^{-1} \varphi \star \partial_{\mu}^{-1} \varphi \star e^{-1}\right)\right\}
\end{aligned}
$$

and

$$
\begin{aligned}
B_{\partial}= & \frac{a}{2 \theta^{2}} \int \mathrm{d}^{d} x\left(\delta_{\varphi^{c}} e\right)\left(\partial_{\mu}^{-1} \varphi_{c} \star \partial_{\mu}^{-1} \varphi^{c} \star e^{-1}\right)+\frac{a}{2 \theta^{2}} \int e \mathrm{~d}^{d} x \delta_{\varphi^{c}}\left(\partial_{\mu}^{-1} \varphi_{c} \star \partial_{\mu}^{-1} \varphi^{c} \star e^{-1}\right) \\
= & \frac{a}{2 \theta^{2}} \int \mathrm{d}^{d} x\left\{\partial_{\sigma}\left(\left(e e_{a}^{\sigma} \delta \varphi^{a}\right) \partial_{\mu}^{-1} \varphi_{c} \star \partial_{\mu}^{-1} \varphi^{c} \star e^{-1}\right)-e e_{a}^{\sigma} \delta \varphi^{a} \partial_{\sigma}\left(\partial_{\mu}^{-1} \varphi_{c} \star \partial_{\mu}^{-1} \varphi^{c} \star e^{-1}\right)\right\} \\
& +\frac{a}{2 \theta^{2}} \int \mathrm{d}^{d} x\left\{-\delta \varphi_{c} \partial_{\mu}^{-1}\left(e\left\{\partial_{\mu}^{-1} \varphi^{c}, e^{-1}\right\}_{\star}\right)+\partial_{\mu}\left(\partial_{\mu}^{-1} \delta \varphi_{c} \partial_{\mu}^{-1}\left(e\left\{\partial_{\mu}^{-1} \varphi, e^{-1}\right\}_{\star}\right)\right)\right. \\
& \left.+2 \partial_{\sigma}\left(e e_{a}^{\sigma} S(\Delta)\left(\partial_{\mu}^{-1} \varphi_{c}, \widetilde{X}^{a} \delta \varphi^{c} \star e^{-1}\right)\right)+\partial_{\sigma}\left(e e_{a}^{\sigma} T(\Delta)\left(\partial_{\mu}^{-1} \delta \varphi_{c}, \widetilde{X}^{a}\left\{\partial_{\mu}^{-1} \varphi^{c}, e^{-1}\right\}_{\star}\right)\right)\right\} \\
& +\frac{a}{2 \theta^{2}} \int e \mathrm{~d}^{d} x\left\{-\delta \varphi^{a} X_{a} \partial_{\mu}^{-1} \varphi_{c}\left\{\partial_{\mu}^{-1} \varphi^{c}, e^{-1}\right\}_{\star}-X_{b} T(\Delta)\left(\delta \varphi^{a} X_{a} \partial_{\mu}^{-1} \varphi_{c}, \widetilde{X}^{b}\left\{\partial_{\mu}^{-1} \varphi^{c}, e^{-1}\right\}_{\star}\right)\right. \\
& -2 X_{b} S(\Delta)\left(\partial_{\mu}^{-1} \varphi_{c}, \tilde{X}^{b} \delta \varphi^{a} X_{a} \partial_{\mu}^{-1} \varphi^{c} \star e^{-1}\right)-X_{a}\left(\partial_{\mu}^{-1} \varphi_{c} \star \partial_{\mu}^{-1} \varphi^{c} \star \delta \varphi^{a} e^{-1}\right) \\
& +\delta \varphi^{a} e^{-1} X_{a}\left(\partial_{\mu}^{-1} \varphi_{c} \star \partial_{\mu}^{-1} \varphi^{c}\right)+X_{b} T(\Delta)\left(X_{a}\left(\partial_{\mu}^{-1} \varphi_{c} \star \partial_{\mu}^{-1} \varphi^{c}\right), \widetilde{X}^{b} \delta \varphi^{a} e^{-1}\right) \\
& \left.+\delta \varphi^{a} X_{a}\left(\partial_{\mu}^{-1} \varphi_{c} \star \partial_{\mu}^{-1} \varphi^{c} \star e^{-1}\right)\right\} .
\end{aligned}
$$


Taking into account all of these quantities, the contribution to the EL equations of motion is

$$
\begin{aligned}
E_{\partial}^{\varphi \varphi^{c}}= & \frac{a}{2 \theta^{2}}\left[-e X_{a} \partial_{\mu}^{-1} \varphi\left\{\partial_{\mu}^{-1} \varphi, e^{-1}\right\}_{\star}+X_{a}\left(\partial_{\mu}^{-1} \varphi \star \partial_{\mu}^{-1} \varphi\right)-e X_{a} \partial_{\mu}^{-1} \varphi_{c}\left\{\partial_{\mu}^{-1} \varphi^{c}, e^{-1}\right\}_{\star}\right. \\
& \left.+X_{a}\left(\partial_{\mu}^{-1} \varphi_{c} \star \partial_{\mu}^{-1} \varphi^{c}\right)-\partial_{\mu}^{-1}\left(e\left\{\partial_{\mu}^{-1} \varphi^{c}, e^{-1}\right\}_{\star}\right)\right] .
\end{aligned}
$$

The contribution to the current $J^{\sigma}$ denoted by $J_{\partial}^{\sigma}$ takes the form

$$
\begin{aligned}
J_{\partial}^{\sigma}= & \frac{a}{2 \theta^{2}}\left[e e_{b}^{\sigma} \delta \varphi^{b} \partial_{\mu}^{-1} \varphi \star \partial_{\mu}^{-1} \varphi \star e^{-1}-e e_{b}^{\sigma} T(\Delta)\left(\delta \varphi^{a} X_{a} \partial_{\mu}^{-1} \varphi, \widetilde{X}^{b}\left(\left\{\partial_{\mu}^{-1} \varphi, e^{-1}\right\}_{\star}\right)\right)\right. \\
& -2 e e_{b}^{\sigma} S(\Delta)\left(\partial_{\mu}^{-1} \varphi, \widetilde{X}^{b} \delta \varphi^{a} X_{a} \partial_{\mu}^{-1} \varphi \star e^{-1}\right)-e e_{b}^{\sigma}\left(\partial_{\mu}^{-1} \varphi \star \partial_{\mu}^{-1} \varphi \star \delta \varphi^{b} e^{-1}\right) \\
& +e e_{b}^{\sigma} T(\Delta)\left(X_{a}\left(\partial_{\mu}^{-1} \varphi \star \partial_{\mu}^{-1} \varphi\right), \widetilde{X}^{b} \delta \varphi^{a} e^{-1}\right)+e e_{b}^{\sigma} \delta \varphi^{b}\left(\partial_{\mu}^{-1} \varphi_{c} \star \partial_{\mu}^{-1} \varphi^{c} \star e^{-1}\right) \\
& +\left(\partial_{\sigma}^{-1} \delta \varphi_{c}\right) \partial_{\sigma}^{-1}\left(e\left\{\partial_{\sigma}^{-1} \varphi^{c}, e^{-1}\right\}_{\star}\right)+2 e e_{b}^{\sigma} S(\Delta)\left(\partial_{\mu}^{-1} \varphi_{c}, \widetilde{X}^{b} \delta \varphi^{c} e^{-1}\right) \\
& +e e_{b}^{\sigma} T(\Delta)\left(\partial_{\mu}^{-1} \delta \varphi_{c}, \widetilde{X}^{b}\left\{\partial_{\mu}^{-1} \varphi^{c}, e^{-1}\right\}_{\star}\right)-e e_{b}^{\sigma} T(\Delta)\left(\delta \varphi^{a} X_{a} \partial_{\mu}^{-1} \varphi_{c}, \widetilde{X}^{b}\left(\left\{\partial_{\mu}^{-1} \varphi^{c}, e^{-1}\right\}_{\star}\right)\right) \\
& -2 e e_{b}^{\sigma} S(\Delta)\left(\partial_{\mu}^{-1} \varphi_{c}, \widetilde{X}^{b} \delta \varphi^{a} X_{a} \partial_{\mu}^{-1} \varphi^{c} \star e^{-1}\right)-e e_{b}^{\sigma}\left(\partial_{\mu}^{-1} \varphi_{c} \star \partial_{\mu}^{-1} \varphi^{c} \star \delta \varphi^{a} e^{-1}\right) \\
& \left.+e e_{b}^{\sigma} T(\Delta)\left(X_{a}\left(\partial_{\mu}^{-1} \varphi_{c} \star \partial_{\mu}^{-1} \varphi^{c}\right), \widetilde{X}^{b} \delta \varphi^{a} e^{-1}\right)\right] .
\end{aligned}
$$

By performing the same computation to the other terms in action (39) we get the EL equations of motion (47) and the current (48), such that

$$
\delta_{\varphi^{c}} \mathcal{S}_{\star}=\int \mathrm{d}^{d} x\left(\delta \varphi^{c} E^{\varphi \varphi^{c}}+\partial_{\sigma} \mathcal{J}^{\sigma}\right)
$$

Open Access This is an open access article distributed under the terms of the Creative Commons Attribution License (http://creativecommons.org/licenses/by/4.0), which permits unrestricted use, distribution, and reproduction in any medium, provided the original work is properly cited.

\section{References}

1. M.R. Douglas, N.A. Nekrasov, Rev. Mod. Phys. 73, 977 (2001) hep-th/0106048.

2. R.J. Szabo, Phys. Rep. 378, 207 (2003) hep-th/0109162.

3. R. Gurau, J. Magnen, V. Rivasseau, F. Vignes-Tourneret, Commun. Math. Phys. 267, 515 (2006) hep-th/0512271.

4. H. Grosse, R. Wulkenhaar, Fortsch. Phys. 53, 634 (2005).

5. H. Grosse, R. Wulkenhaar, Commun. Math. Phys. 256, 305 (2005) hep-th/0401128.

6. D. D'Ascanio, P. Pisani, D.V. Vassilevich, Eur. Phys. J. C 76, 180 (2016) arXiv:1602.01479 [hep-th].

7. A. Tanasa, SIGMA 6, 047 (2010) arXiv:1003.4877 [hep-th].

8. A. Tanasa, P. Vitale, Phys. Rev. D 81, 065008 (2010) arXiv:0912.0200 [hep-th].

9. R. Gurau, J. Magnen, V. Rivasseau, A. Tanasa, Commun. Math. Phys. 287, 275 (2009) arXiv:0802.0791 [math-ph].

10. A. Tanasa, Rom. J. Phys. 53, 1207 (2008) arXiv:0808.3703 [hep-th].

11. A. Tanasa, J. Phys. A 42, 365208 (2009) arXiv:0807.2779 [math-ph].

12. S. Doplicher, K. Fredenhagen, J.E. Roberts, Commun. Math. Phys. 172, 187 (1995) hep-th/0303037.

13. S. Majid, Lect. Notes Phys. 541, 227 (2000) hep-th/0006166.

14. P. Aschieri, L. Castellani, Gen. Relativ. Gravit. 45, 411 (2013) arXiv:1206.4096 [hep-th].

15. P. Aschieri, M. Dimitrijevic, P. Kulish, F. Lizzi, J. Wess, Lect. Notes Phys. 774, 1 (2009).

16. P. Aschieri, L. Castellani, M. Dimitrijevic, Lett. Math. Phys. 85, 39 (2008) arXiv:0803.4325 [hep-th].

17. P. Aschieri, J. Phys. Conf. Ser. 53, 799 (2006) hep-th/0608172.

18. P. Aschieri, M. Dimitrijevic, F. Meyer, J. Wess, Class. Quantum Grav. 23, 1883 (2006) hep-th/0510059.

19. M.N. Hounkonnou, D.O. Samary, J. Math. Phys. 51, 102108 (2010) arXiv:1008.1325 [math-ph].

20. M.N. Hounkonnou, D.O. Samary, J. Phys. A 44, 315401 (2011).

21. A. Micu, M.M. Sheikh Jabbari, JHEP 01, 025 (2001) hep-th/0008057.

22. E. Langmann, R.J. Szabo, Phys. Lett. B 533, 168 (2002) hep-th/0202039.

23. J. Ben Geloun, M.N. Hounkonnou, Phys. Lett. B 653, 343 (2007).

24. J. Ben Geloun, M.N. Hounkonnou, AIP Conf. Proc. 956, 55 (2007).

25. M.N. Hounkonnou, D.O. Samary, AIP Conf. Proc. 1079, 82 (2008) arXiv:1203.5564 [math-ph].

26. M.N. Hounkonnou, D.O. Samary, J. Phys. A 43, 155202 (2010) arXiv:0909.4562 [math-ph].

27. J. Ben Geloun, A. Tanasa, Lett. Math. Phys. 86, 19 (2008) arXiv:0806.3886 [math-ph].

28. V. Rivasseau, From Perturbative to Constructive Renormalization (Princeton University Press, Princeton, 1991). 
29. A. Gerhold, J. Grimstrup, H. Grosse, L. Popp, M. Schweda, R. Wulkenhaar, The energy momentum tensor on noncommutative spaces. Some pedagogical comments, hep-th/0012112.

30. M. Abou-Zeid, H. Dorn, Phys. Lett. B 514, 183 (2001) hep-th/0104244.

31. R. Jackiw, Dynamical Symmetry Breaking, MIT-CTP-403.

32. C.G. Callan Jr., S.R. Coleman, R. Jackiw, Ann. Phys. 59, 42 (1970).

33. S.R. Coleman, R. Jackiw, Ann. Phys. 67, 552 (1971).

34. S. Ghosh, Space-time symmetries in noncommutative gauge theory: A Hamiltonian analysis, hep-th/0310155.

35. A.K. Das, J. Frenkel, Phys. Rev. D 67, 067701 (2003) hep-th/0212122.

36. J.M. Grimstrup, B. Kloibock, L. Popp, V. Putz, M. Schweda, M. Wickenhauser, Int. J. Mod. Phys. A 19, 5615 (2004) hep-th/0210288.

37. H. Balasin, D.N. Blaschke, F. Gieres, M. Schweda, Eur. Phys. J. C 75, 284 (2015) arXiv:1502.03765 [hep-th].

38. T. Nakamura, S. Hamamoto, Prog. Theor. Phys. 95, 469 (1996) hep-th/9511219.

39. M. Ostrogradski, Mem. Ac. St. Petersbourg V14, 385 (1850).

40. K. Bering, A note on nonlocality and Ostrogradski's construction, hep-th/0007192.

41. J. Gomis, K. Kamimura, J. Llosa, Phys. Rev. D 63, 045003 (2001) hep-th/0006235. 